\title{
ANALYSING THE IMPACT ON UNDERWATER NOISE OF CHANGES TO THE PARAMETERS OF A SHIP'S MACHINERY
}

\author{
Krystian Buszman \\ Polish Naval Academy, Poland
}

\begin{abstract}
A ship moving over the surface of water generates disturbances that are perceived as noise, both in the air and under water. Due to its density, water is an excellent medium for transmitting acoustic waves over long distances.

This article describes the impact of the settings of a ship's machinery on the nature of the generated noise. Our analysis includes the frequency characteristics of the noise generated by the moving ship. Data were obtained using an underwater measurement system, and the measured objects were two ships moving on specific trajectories with certain machinery settings. The acquired data were analysed in the frequency domain to explore the possibilities of the acoustic classification of ships and diagnostics of source mechanisms.
\end{abstract}

Keywords: shipping noise, hydroacoustic passive measurement, narrowband, 1/3-octave analysis, diagnosis, classification

\section{INTRODUCTION}

The monitoring of underwater noise generated by ships has been a topic of interest to research scientists for several years $[11,14]$, and measurement standards have been developed for this type of noise [10]. One area of research is the impact of noise on the natural environment $[2,12]$, and in particular on underwater fauna, which researchers from many institutes and pro-ecological organisations have focused on $[3,15]$. Another aspect is mechanical engineering methods of diagnostics for ship equipment $[9,13]$, using on-board and external measurement systems. A further area of underwater noise research is the safety of the ship in terms of the threat from non-contact mines with hydroacoustic igniters [5, 6]. In addition, studies have been conducted for statistical purposes for different types of customers, for example physicists, biologists, logisticians and various services connected with maritime transport. In each of these areas, the parameters characterising the recorded underwater noise are very important. The noise emanating from ships is connected with the equipment necessary for their movement, which uses specific parameters. The main parameter is the speed, which determines the attributes of the ship's machinery of operation. A change in a ship's propulsion settings affects the speed of the ship, and causes noise with different characteristics to be generated. 


\section{DISTINGUISHABILITY OF OBJECTS BASED ON THE PARAMETERS OF MECHANICAL OPERATION OF SHIPS}

An underwater noise analysis was carried out based on measurements of a particular ship using a bottom measuring system $[6,7]$. The measuring circuit included a hydrophone that received signals in the range $5-120 \mathrm{kHz}$. The complete measuring system consisted of a recorder unit with $\mathrm{A} / \mathrm{D}$ converters with a maximum sampling frequency of $51.2 \mathrm{kHz}$, and a computer with data logging software. The recorder unit was located inside the bottom nod, which was connected with the computer at a land-based station via a hybrid cable. A block diagram of this system is shown in Fig. 1.

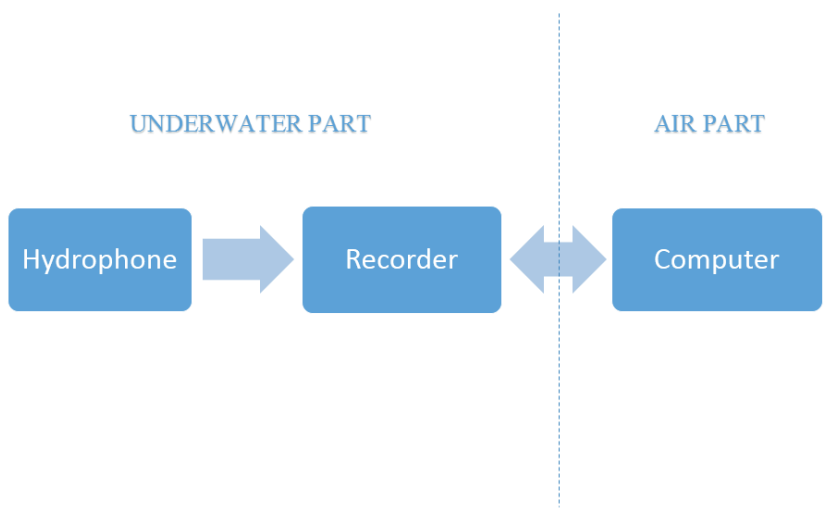

Fig. 1. Block diagram of the underwater noise measurement circuit.

The input parameters of the recorded signals determine the output parameters in the frequency domain, and in particular, the frequency resolution depends on the duration of the analysed signal. The width of the overlap was $50 \%$ of the number of samples. This operation allowed us to obtain a set of results as a series of amplitude values for each second of the analysed signal, assuming the determined time for a single analysis.

The results of the signal analysis over the time duration, corresponding to the noise generated by the ship when it passes the hydrophone at its closest point of approach (CPA), are in the form of a spectrum of pressure levels with reference to $1 \mu \mathrm{Pa}$, with a resolution depending on the parameters of the FFT analysis. In case of $50 \%$ overlap, a spectrum was presented for $2 \mathrm{~s}$ of average SPL (102,400 samples). To increase the performance of the FFT analysis, the length of the signal was increased up to the next power of two of the number of samples used $(131,072)$. The resulting frequency resolution was better than $0.5 \mathrm{~Hz}$, as can be seen from the narrowband spectra results.

We also show that it is possible to identify sources of noise on the ship when the RPM of the machinery is known. This depends on the parameters of operation of the ship's machinery. Data were collected onboard during underwater noise measurements. The parameters must be unchanged throughout the signal analysis period. In the following, the spectra for two ships crossing over the hydrophone are shown.

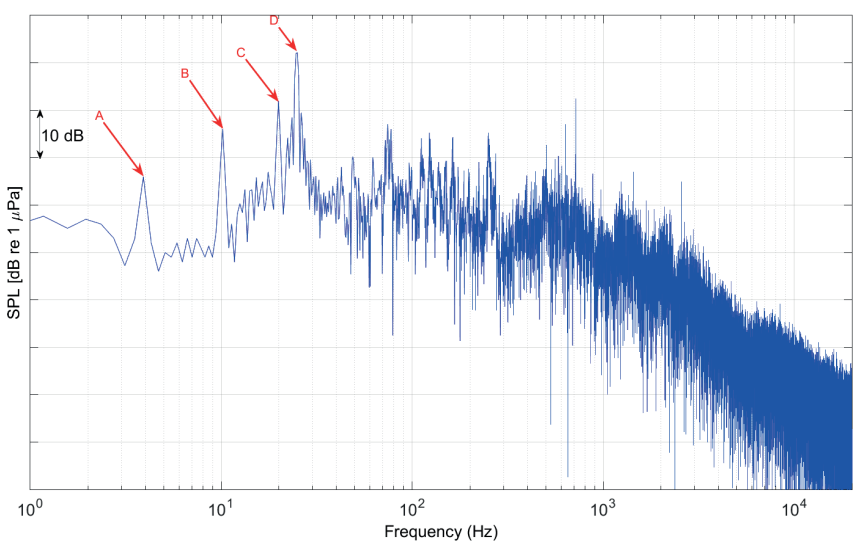

Fig. 2. Narrowband spectrum with specific frequencies corresponding to the working parameters of selected components of the first ship.

The spectrum is the result of a 2 s signal when the ship was at its CPA to the bottom nod in shallow water.

In a document describing the measurement standards for noise generated by ships [10], it is advised to use a longer averaging time for deep water measurements.

Fig. 2 shows several characteristic frequencies related to the first vessel. These are related to the operational components of the ship, which was equipped with two $700 \mathrm{~kW}$ diesel engines and moved with the following parameters, as labelled in Fig. 2: A - drive shafts, rotational speed 234 RPM, $\mathrm{B}$ - main engines, rotational speed $600 \mathrm{RPM}$, C - propellers with five blades, rotational speed 234 RPM, D - generators, rotational speed 1500 RPM.

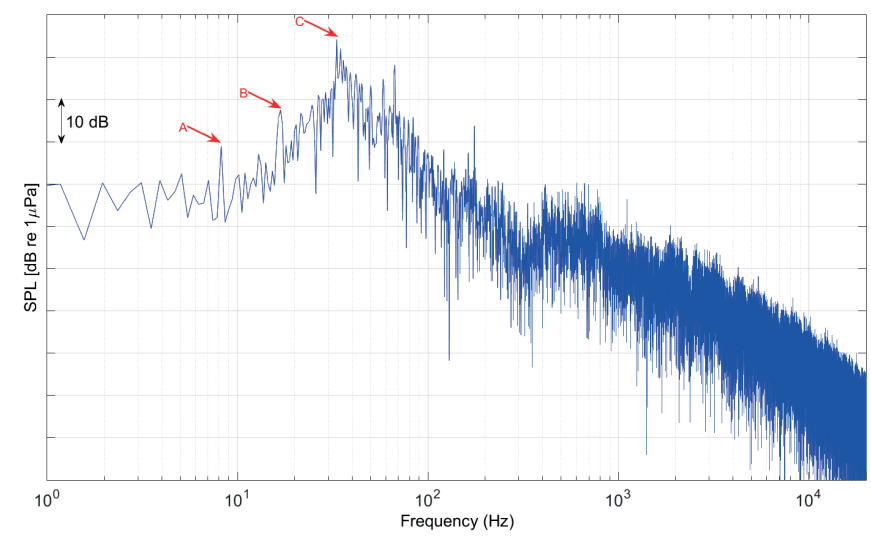

Fig. 3. Narrowband spectrum with the specific frequencies corresponding to the working parameters of selected components of the second ship. The spectrum is based on a 2 signal when the ship was at its CPA to bottom nod in shallow water.

Fig. 3 shows a similar spectrum labelled with the specific characteristic frequencies of the second ship. This ship was equipped with two $9000 \mathrm{~kW}$ diesel engines and the following parameters of motion:

A - main engines, rotational speed 500 RPM,

B - propellers with four blades, rotational speed 252 RPM,

C - generators, rotational speed 1992 RPM. 
By comparing these two figures, differences can be observed in the tonal frequencies and amplitudes, corresponding to differences in the RPM of the machinery. The tonal frequencies are characteristic of a specific vessel at a specific speed; when the speed of the vessel changes, the RPM of the shaft and propulsion engines also changes, while the RPM of the auxiliary engines usually remains constant.

In the frequency spectra presented here for these two ships, there are many other distinctive frequencies associated with other mechanisms of operation. The distinction of selected frequencies is only informative, and offers the possibility of identifying a given ship based on the characteristic frequency components of the recorded signal.

\section{EXPERIMENTAL EXAMINATION OF UNDERWATER NOISE GENERATED BY A MOVING SHIP}

The aim of this research was to determine whether two configurations of a given ship could be distinguished by means of hydroacoustic passive measurements, using repeated passes of the ship over the measurement system.

The ship (length $60 \mathrm{~m}$, width $10 \mathrm{~m}$, draught $3 \mathrm{~m}$ ) had a speed of up to 12 knots, and moved in both directions along a given course above a stationary measuring system. A hydrophone was placed at a depth of about $10 \mathrm{~m}$. The experiment was divided into two stages, each of which required the ship to have different configuration of fixed machinery and speed.

Fig. 4 shows the first set of paths of the ship, in the form of GPS traces plotted on a map of the area used for research.

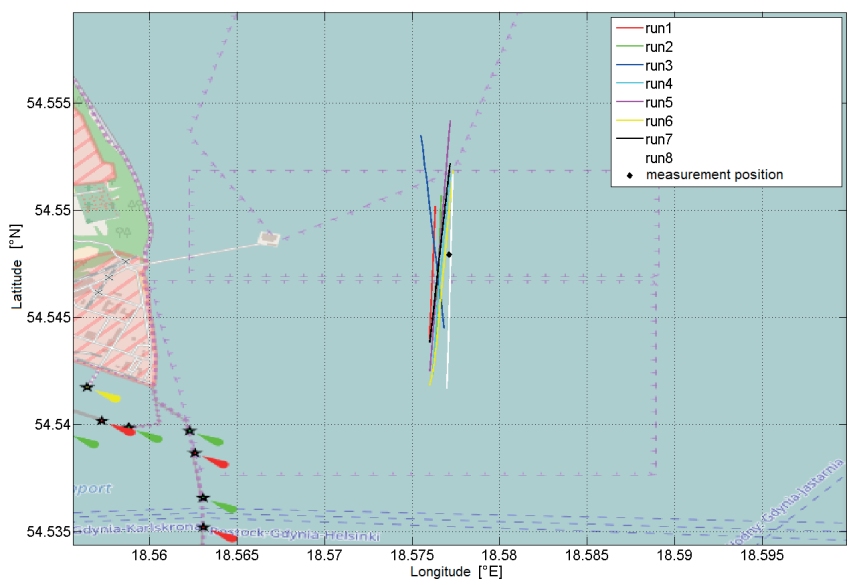

Fig. 4. Map of the area with the first set of trajectories and the position of the measuring system.

Fig. 5 shows the second set of transitions, in which the GPS traces of the ship are shown in the same way.

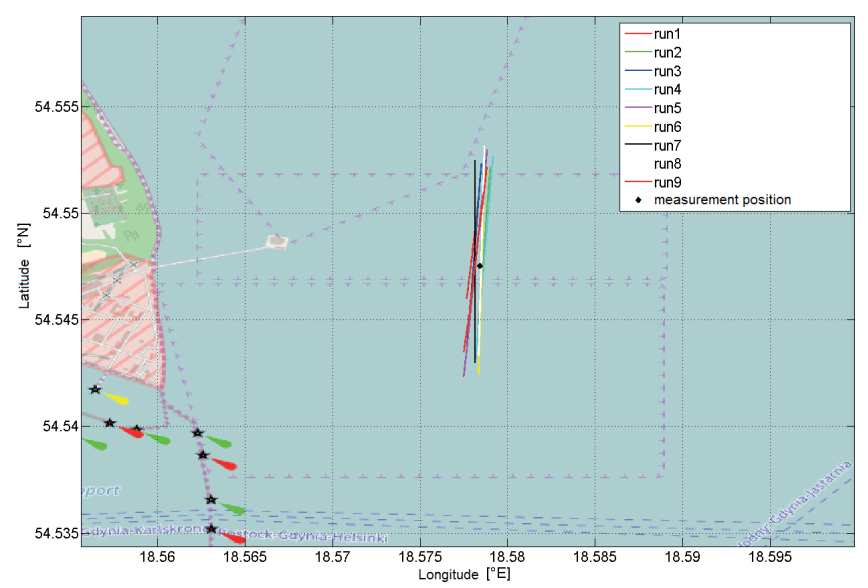

Fig. 5. Map of the area with the second set of trajectories and the position of the measuring system.

The data analysis process was divided into two stages. The first step was to determine the sound pressure level for each of the transitions. Figs. 6 and 7 show the results plotted as 3D charts, showing the distance of the ship from the measurement system.

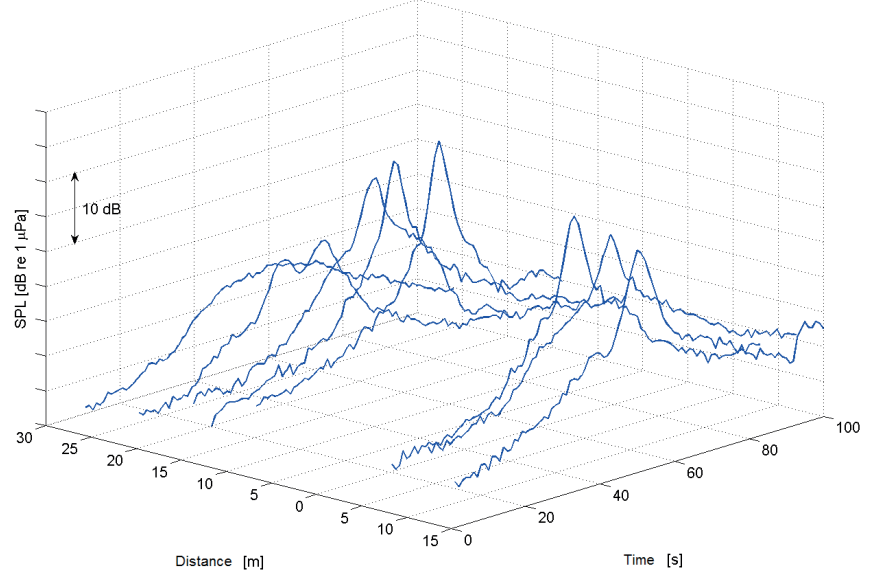

Fig. 6. Set of broadband SPL values for individual ship runs for the first configuration. Results corrected with absorption coefficient in bandwidth up to $25 \mathrm{kHz}$.

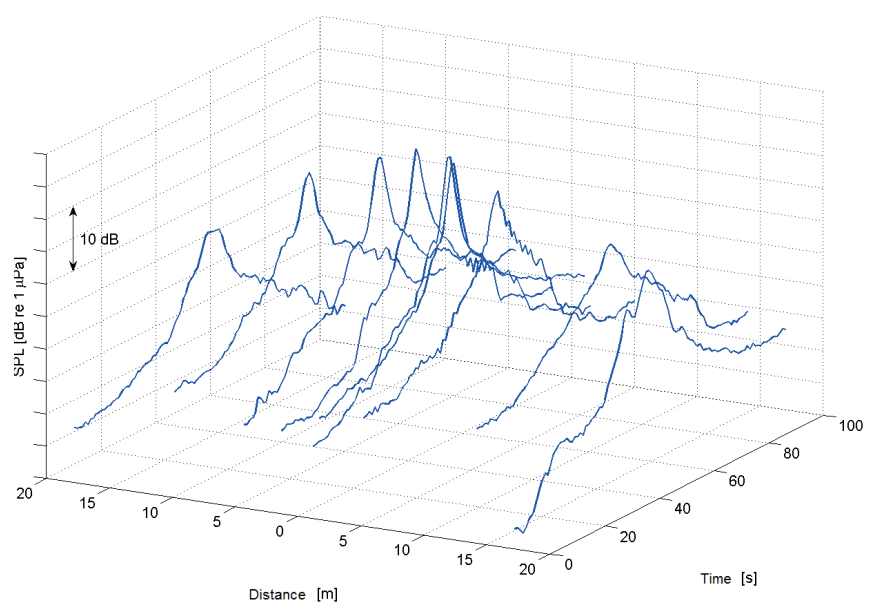

Fig. 7. Set of broadband SPL values for individual ship runs for the second configuration. Results corrected with absorption coefficient in bandwidth up to $25 \mathrm{kHz}$. 
The graphs of SPL show changes (decreases) with increasing distance, both before and after the measuring point along the trajectory of the tested ship. The effect of the lateral distance at which the ship passes the position of the hydrophone can also be observed. The machinery parameters for each configuration were unchanged during each set of runs. CPA data were used at the frequency analysis stage, taking into account the dependence on absorption coefficient as a function of frequency $[1,4]$. The results were corrected using a curve representing absorption in the frequency domain taken from [1], based on the Francois-Garrison equation. According to this equation, the absorption coefficient affects only higher frequencies of the broadband signal, above $1 \mathrm{kHz}$. It is caused by small distances (up to $30 \mathrm{~m}$ ). The absorption loss below $1 \mathrm{kHz}$ is negligible and does not affect the results of measurement. The absorption in different underwater environments is shown in Fig. 8 as a set of curves in the frequency domain up to $10^{3} \mathrm{kHz}[1]$.

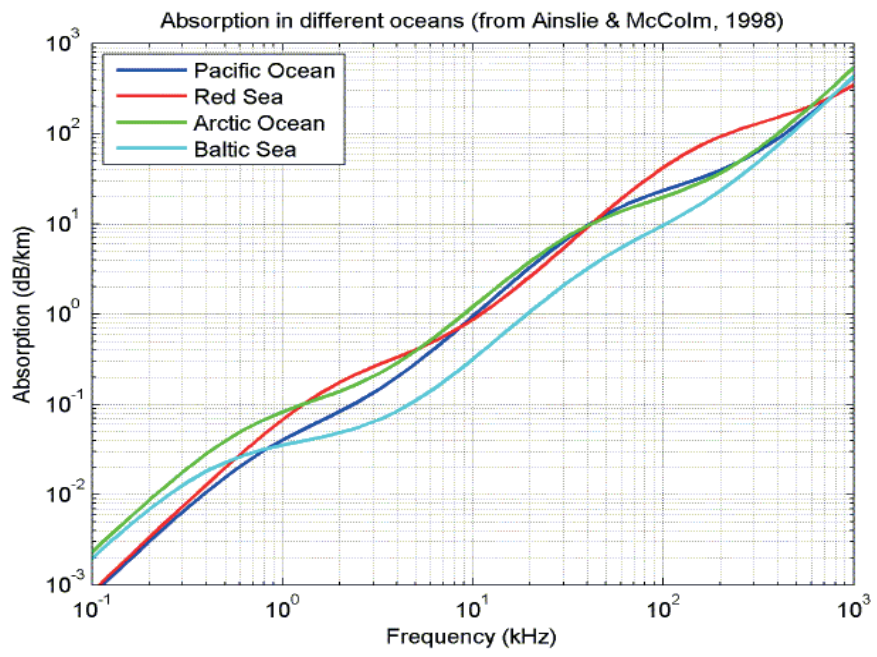

Fig. 8. Acoustic wave absorption versus frequency for different water environments [14].

The next step was to perform a frequency analysis at two bandwidths, 1/3-octave [8] and narrowband. The results of $1 / 3$-octave analysis for each of the transitions are shown as a set of curves in Figs. 9 and 10 for both sets of measurements.

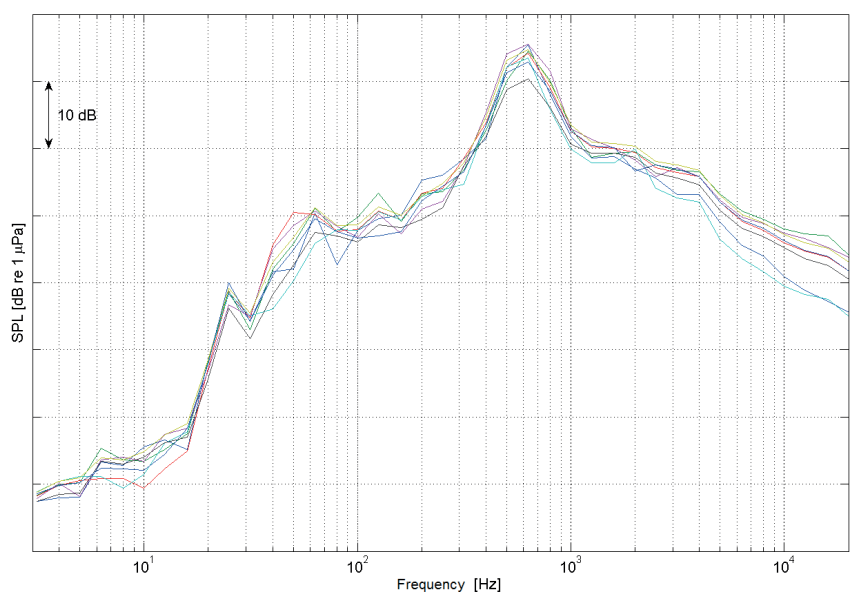

Fig. 9. Results of 1/3-octave spectral analysis for the first configuration with absorption loss correction.

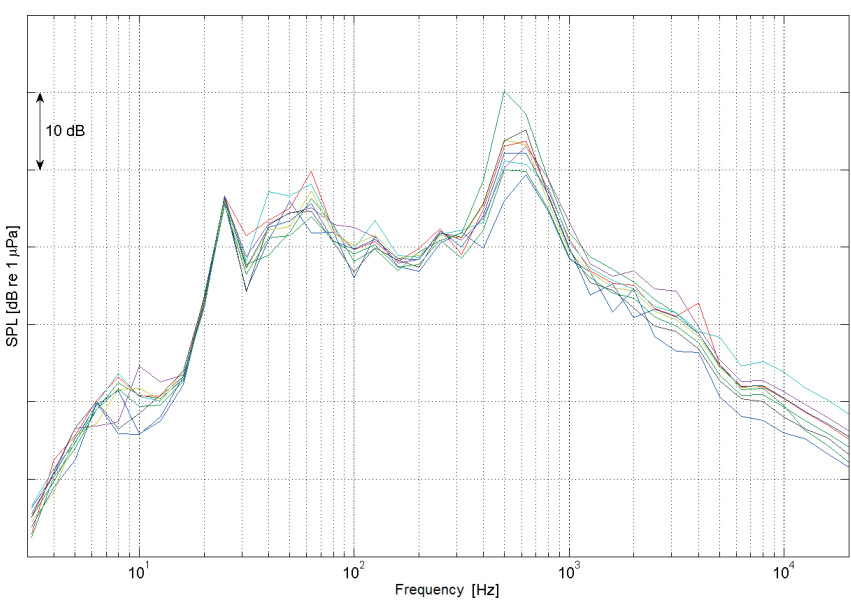

Fig. 10. Results of 1/3-octave spectral analysis for the second configuration with absorption loss correction.

The results of 1/3-octave analysis shown in Figs. 9 and 10 were obtained for two configurations of the ship's machinery, with repeated measurements. The narrowband spectrum was analysed in the same way.

To demonstrate the differences in the spectra for the two configurations of the ship's machinery, the result for all runs were averaged and presented in Fig. 11.

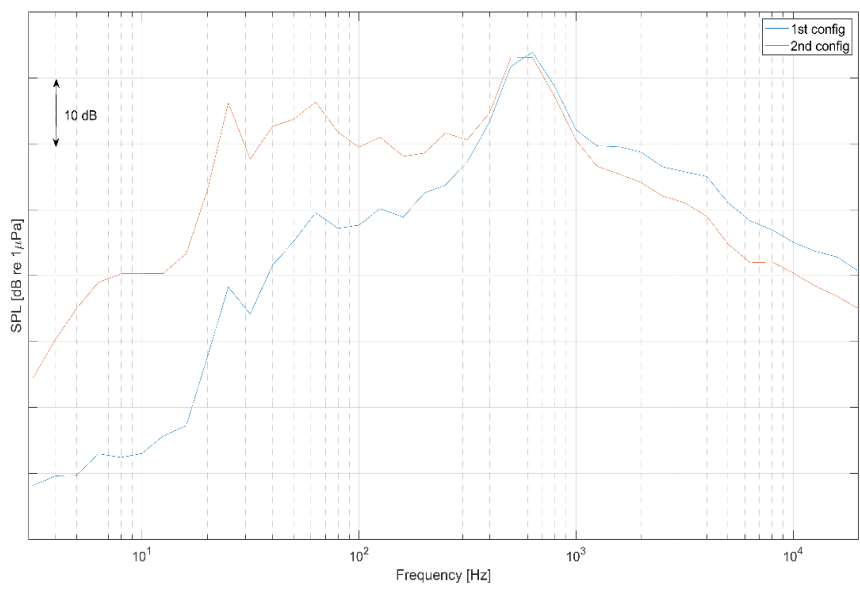

Fig. 11. Comparison between the average results of a 1/3-octave spectral analysis for two configurations of the operating mechanism of a ship.

A narrowband frequency analysis was performed in the range $5-2 \mathrm{kHz}$. The spectrum was calculated for the CPA, i.e. the highest SPL value of the signal. The maximum SPL value was located close to the centre of the ship, a few metres to the stern. This analysis also takes into account the absorption coefficient as a function of frequency, based on the Francois-Garrison equation. A set of curves was obtained representing the amplitude spectra for the same ship for the two configurations, and the results are shown in Figs. 12 and 13. 


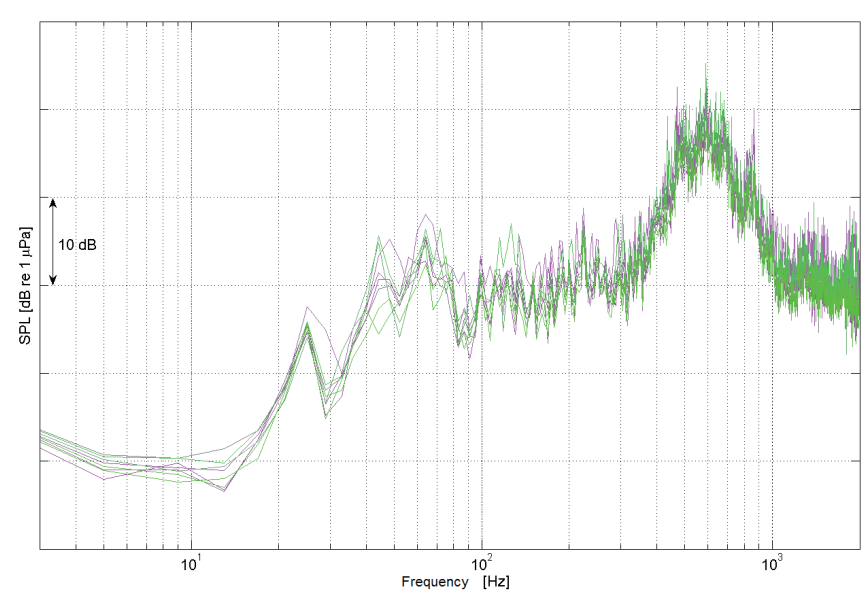

Fig. 12. Results of narrowband spectral analysis for the first configuration.

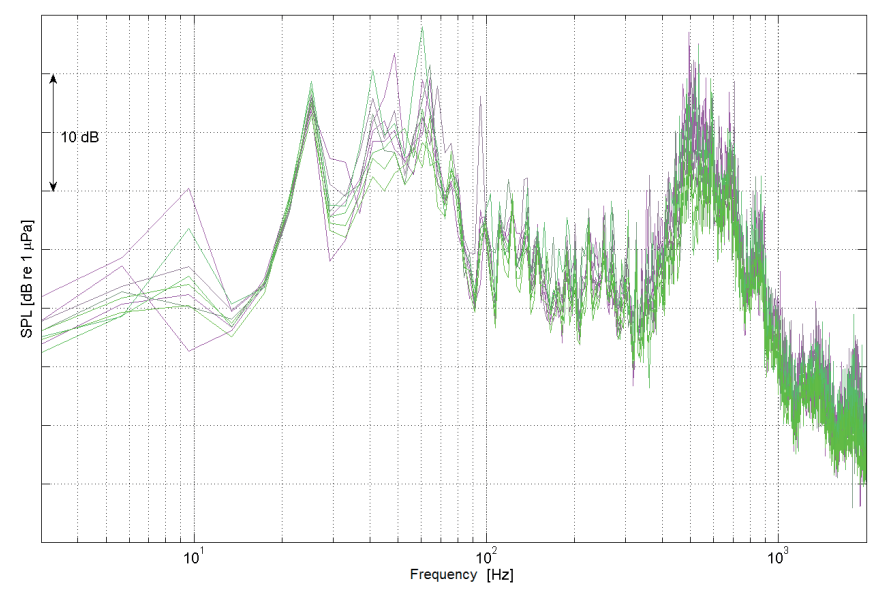

Fig. 13. Results of narrowband spectral analysis for the second configuration.

In a similar way to the $1 / 3$-octave spectrum analysis, the narrowband spectrum results for all runs were averaged for each configuration, and are shown in Fig. 14. Both these results and those for the $1 / 3$-octave spectrum show how the noise spectra generated by a given ship are connected with the configuration of the source parameters for the ship's machinery. This demonstrates the possibility of applying diagnostics to a particular configuration for a ship.

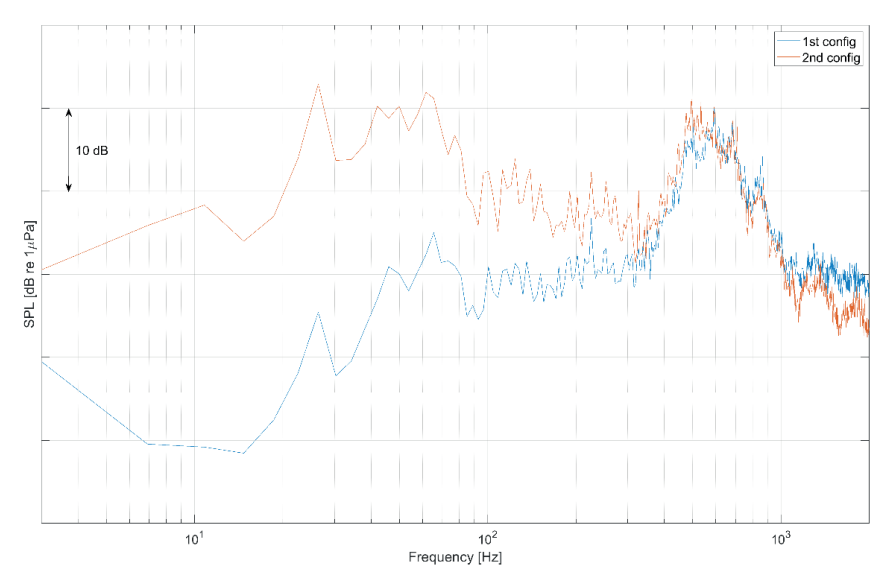

Fig. 14. Comparison between average narrowband spectral analysis results for two configurations of a ship's operating mechanism.
The sound pressure level of between $300 \mathrm{~Hz}$ and $1 \mathrm{kHz}$ was similar for the first and second configurations, suggesting that the predominant source mechanism does not change when the configuration changes. A similarity between the amplitude spectra for both configurations can be observed; characteristic frequencies appear in both cases, although the amplitudes differ. This means that a given type of a ship could be identifiable based on the specific frequencies, while the differences in level indicate a change in the parameters of the ship's components.

All spectra shown in Figs. 9 to 14 were corrected for the absorption effect. The absorption calculations were made for the near field, and were based on the Francois-Garrison formula for the relevant bandwidth, for a water depth of up to $10 \mathrm{~m}$ with salinity $7 \mathrm{ppm}$. There were no significant differences in the results obtained for the different directions in which the ship passed, but the heading data were collected for each pass and added as metadata to the recorded signals.

\section{CONCLUSIONS}

A frequency analysis of the hydroacoustic field of a moving ship can be used to support the process of object classification. These analyses can also be used in diagnostic testing of a ship's machinery in conjunction with the use of an on-board measurement system. To achieve this, it is necessary to create a database of the main settings and operating parameters of the ship's equipment. For ships of the same type, it is possible to determine the configuration of the operating equipment based on the narrowband and 1/3-octave spectra.

The use of an experiment involving multiple transitions of the ship with certain settings demonstrated similarities between the designated individual spectra for CPA.

\section{REFERENCES}

1. Ainslie M. A., McColm J. G. (1998): A simplified formula for viscous and chemical absorption in sea water. The Journal of the Acoustical Society of America, 103(3), 1671-1672. DOI: $10.1121 / 1.421258$.

2. Arveson P. T., Vendittis D. J. (2000): Radiated noise characteristics of a modern cargo ship. The Journal of the Acoustical Society of America, 107(1), 118-129. DOI: $10.1121 / 1.428344$.

3. Erbe C., Marley S. A., Schoeman R. P., Smith J. N., Trigg L. E., Embling C. B. (2019): The effects of ship noise on marine mammals. Review Frontiers in Marine Science, 6. DOI: 10.3389/fmars.2019.00606.

4. Francois R. E., Garrison G. R. (1982): Sound absorption based on ocean measurements: Part II: Boric acid contribution and equation for total absorption. Journal of the Acoustical Society of America, 72(6), 1879-1890. DOI: 10.1121/1.388673. 
5. Freitag L., Grund M., Von Alt C., Stokey R. (2005): A shallow water acoustic network for mine countermeasures operations with autonomous underwater vehicles. Conference Proceedings of Underwater Defense.

6. Gloza I., Buszman K. (2011): The multi-influence passive module for underwater environment monitoring. Hydroacoustics, 14, 47-54.

7. Gloza I., Buszman K., Józwiak R. (2013): Tracking underwater noise sources with the use of a passive method. Acta Physica Polonica A, 123(5), 1090-1093. DOI: 10.12693/ APhysPolA.123.1090.

8. Gloza I., Józwiak R., Buszman K. (2014): The one-third octave spectrum as a method of vessel identification. Hydroacoustics, 17, 63-68.

9. Grządziela A. (2006): Analysis of vibration parameters of ship gas turbine engines. Polish Maritime Research, 2, 22-26.

10. ISO 17208-1:2016 (2016): Underwater acoustics-Quantities and procedures for description and measurement of underwater sound from ships.

11. Kozaczka E., Grelowska, G. (2018): Propagation of shipgenerated noise in shallow sea. Polish Maritime Research, 2(98), 37-46. DOI: 10.2478/pomr-2018-0052.

12. Kuşku H., Yiğit M., Ergün S., Yiğit Ü., Taylor N. (2018): Acoustic noise pollution from marine industrial activities: Exposure and impacts. Aquatic Research, 1(4), 148-161. DOI: $10.3153 / A R 18017$.

13. Lus T. (2012): Marine diesel engine diagnostics in operating conditions. Diagnostyka, 2(62), 43-47.

14. McKenna M. F. (2012): Underwater radiated noise from modern commercial ships. Journal of the Acoustical Society of America, 131(1), 92-103. DOI: 10.1121/1.3664100.

15. Southall B. L. (2004): Shipping noise and marine mammals: A forum for science, management, and technology. Final Report of the National Oceanic and Atmospheric Administration (NOAA) International Symposium. U.S. NOAA Fisheries, Arlington, Virginia, May 18-19.

\section{CONTACT WITH THE AUTHOR}

Krystian Buszman

e-mail:k.buszman@amw.gdynia.pl

Polish Naval Academy,

Smidowicza 69

81-127 Gdynia,

POLAND 